\title{
Electrical-contact-free readout of the response of superconductive bolometer arrays using thermal cross talk
}

\author{
Ali Bozbey, Mehdi Fardmanesh, Juergen Schubert, and Marko Banzet
}

\begin{abstract}
We utilized and investigated the unique dependence of the magnitude and phase of the response on thermal cross talk between bolometer pixels in an array to measure the response of the devices through fewer monitoring devices. We show the feasibility of the proposed readout technique by use of two source pixels in an array, as the image-mapping devices, and one optically shielded pixel as the readout device. While the sensing pixels were electrical-contact free, the readout device was current biased in 4-probe current-bias configuration. Both the phase and the magnitude of the response due to the cross talk in the array were found to be strongly dependent on the modulation frequency and the distance between the sensing and the readout pixels. A series of measurements were designed to extract the response of each single-sensing pixel. By combining the measured data, the response of individual pixels could be extracted through the interpolation of the mapped responses. (C) 2006 Optical Society of America
\end{abstract}

OCIS codes: $\quad 040.3060,040.1240$.

\section{Introduction}

We have already investigated and reported the parameters that affect the cross talk between neighboring pixels of the bolometer arrays elsewhere. ${ }^{1}$ In Ref. 1 we defined the cross-talk-free modulation frequency $\left(f_{m}\right)$ for operation of the devices in a conventional configuration. That is, each pixel has electrical contacts, and it is favorable that the pixels do not have any cross talk. Since a very large area on most of the detector chips is occupied by readout electronics and/or contact paths, it is favorable to decrease the electrical contact areas or contacts made to the sensor pixels. Decrease of the electrical contacts when possible would lead to denser layout designs that enable increased spatial resolution and decrease the power consumption and the fabrication cost. In this study, we operate the devices below their cross-talk-free $f_{m}$ to utilize the cross talk between the devices in an

A. Bozbey (bozbey@ieee.org) and M. Fardmanesh are with the Department of Electrical and Electronics Engineering, Bilkent University, Ankara 06800, Turkey. J. Schubert and M. Banzet are with ISG1-IT and Center of Nanoelectronic Systems for Information Technology, Forschungszentrum Juelich GmbH, Juelich D-52425, Germany.

Received 17 February 2006; accepted 12 May 2006; posted 24 May 2006 (Doc. ID 68199).

0003-6935/06/287235-04\$15.00/0

C 2006 Optical Society of America array in detection of the response of the radiation sensing pixels with no electrical contacts. This is done by measuring the phase and magnitude of a read-out device in the array, which is biased using its electrical contacts. To the best of our knowledge, including semiconductor and superconductor detectors, this is the first time that such a readout methodology is proposed and utilized. This approach would allow only one readout pixel to be used for a number of sensing pixels.

To investigate the feasibility of the proposed readout methodology in this study, a design is implemented where a readout pixel is used to read the response of two neighboring detector sensing pixels. We have experimentally found and formulated the unique dependencies of the phase and magnitude of the thermal cross-talk-based responses that enable the measurement of the response of the sensing pixels by fewer monitoring or readout pixels. Here, we show the feasibility of the proposed method and present the design optimizations in terms of device dimensions and operating frequency.

\section{Samples and Experimental Setup}

The cross-talk study was made possible through the illumination of sensing devices and measurement of the voltage response of the blocked readout device in the same array. This was done using a gold-coated shadow mask. To prevent thermal artifacts created 


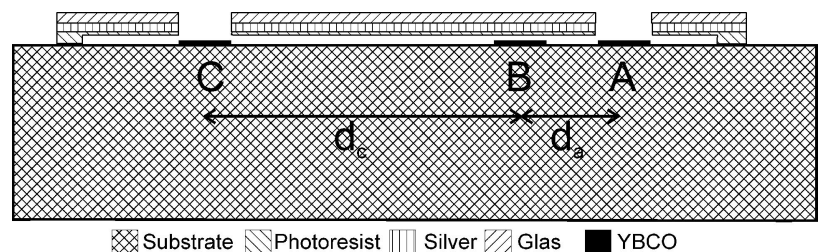

Fig. 1. Side view of the bolometer array. Readout pixel B and sensing pixels $\mathrm{A}$ and $\mathrm{D}$ are shown.

by the mask, the mask was made in a free-standing configuration on top of the devices as shown in Fig. 1. The devices were made of $200 \mathrm{~nm}$ thick pulsed laser-deposited $\mathrm{YB}_{2} \mathrm{C}_{3} \mathrm{O}_{7-x}$ films on $\mathrm{SrTiO}_{3}$ substrate material. The details of the experimental setup and the devices were explained elsewhere. ${ }^{1}$

\section{Results and Discussion}

A. Feasibility of Electrical-Contact-Free Measurement of the Response of Superconductive Bolometer Arrays

We utilized and investigated the unique dependence of the magnitude and phase of the response on the thermal cross talk between bolometer pixels in an array to measure the response of the devices through fewer monitoring devices. Here we show the feasibility of the proposed readout technique by use of two source pixels in an array as the image-mapping devices and one optically shielded pixel as the readout device. While the sensing pixels were electricalcontact free, the readout device was current biased in a 4-probe current-bias configuration. Both the phase and the magnitude of the response due to the cross talk in the array were found to be strongly dependent on the modulation frequency and the distance between the sensing and the readout pixels. A series of measurements were designed to extract the response of each single-sensing pixel. By combining the measured data, the response of individual pixels could be extracted through the interpolation of the mapped responses.

To test the feasibility of the proposed detection mechanism, we have implemented the device array configuration as shown in Fig. 1. The readout pixel is chosen as device $\mathrm{B}$, whereas devices $\mathrm{A}$ and $\mathrm{C}$ were chosen to be the sensing pixels being exposed to the incident radiation. Device B has contacts for 4-probe measurements, and devices $\mathrm{A}$ and $\mathrm{C}$ do not have any electrical contacts. The goal of this study is to find the methodology for extracting the response of the A and $\mathrm{C}$ pixels through the measured signal of the currentbiased device $\mathrm{B}$.

\section{B. Principle of Operation}

The spatial and frequency dependence of the response at distance $x$ away from a single pixel bolometer has been formulated $\mathrm{as}^{2,3}$

$$
\frac{T(x, f)}{T_{0}}=\underbrace{\exp \left(-\sqrt{\frac{\pi f}{D} x}\right)}_{\text {Magnitude }} \exp \underbrace{\left(-\sqrt{\frac{\pi f}{D} x j}\right)}_{\text {Phase }},
$$

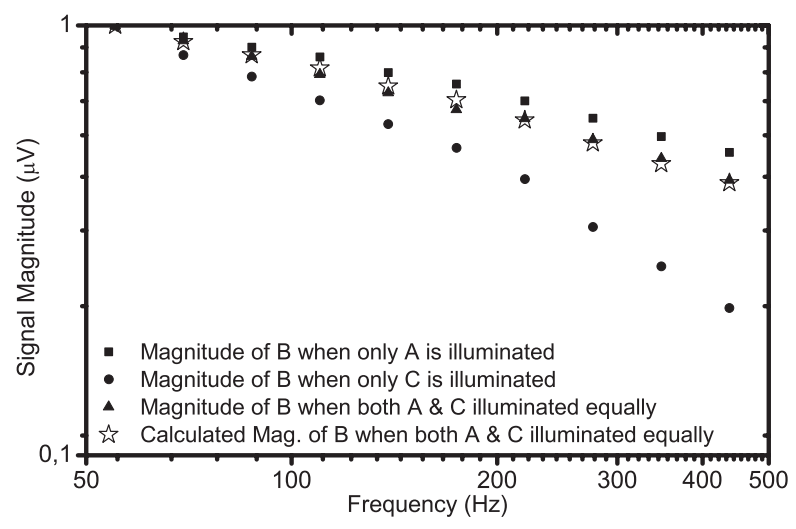

(a)

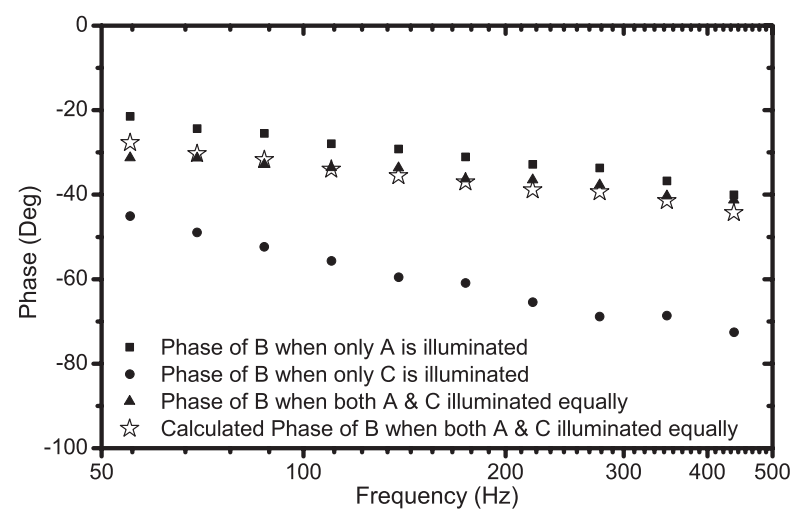

(b)

Fig. 2. (a) Response magnitudes and (b) phases of B under various illuminations of $\mathrm{A}$ and $\mathrm{C}(\boldsymbol{\square}, \bullet$, and $\mathbf{\Delta})$. The magnitude and phase of the sum of the cross-talk responses of A and C ( $\star$ ) fit to that of the phase and magnitude of $\mathrm{B}(\mathbf{\Delta})$.

where $T(x, f) / T_{0}$ is the normalized response under modulation frequency of $f$ at $x$ distance away from the source pixel, and $D$ is the diffusivity of the substrate material. As shown in Eq. (1), with the increase of the distance $x$, the phase of the response, $-\sqrt{\pi f / D} x$, decreases resulting in a further increase of the lag of the signal.

Considering the cross talk for a 2-pixel case, the cross-talk response at point $x_{b}$, caused by devices $\mathrm{A}$ and $\mathrm{C}$, are the superposition of the responses of individual pixels $\mathrm{A}$ and $\mathrm{C}$ at $x_{b}$. Equation (2) shows this superposition relation

$$
\begin{aligned}
\frac{T\left(x_{b}, f\right)}{T_{0}}= & \exp \left(-\sqrt{\frac{\pi f}{D}} d_{a}\right) \exp \left(-j \sqrt{\frac{\pi f}{D}} d_{a}\right) \\
& +\exp \left(-\sqrt{\frac{\pi f}{D}} d_{c}\right) \exp \left(-j \sqrt{\frac{\pi f}{D}} d_{c}\right)
\end{aligned}
$$

where $d_{a}$ and $d_{c}$ are the distances of $\mathrm{A}$ and $\mathrm{C}$ pixels from the $\mathrm{B}$ pixel, respectively. Figure 1 shows the implemented design and the structure of the array with the shadow mask. In Fig. 2, the superposition relation is shown together with experimental data confirming the validity of the above superposition 
(due to the interference) assumption for $x_{a}=40 \mu \mathrm{m}$ and $x_{c}=170 \mu \mathrm{m}$. The readout pixel $\mathrm{B}$ will have a response due to sensing pixels $\mathrm{A}$ and $\mathrm{C}$ with $x$ $=40$ and $170 \mu \mathrm{m}$, respectively. If we add these two responses in vector form to include both their phase and magnitude contributions, we get the curves denoted by ( $\mathbf{\Delta})$ in Fig. 2. The three experimental curves in Fig. 2 are obtained by three illumination configurations as (i) only A, (ii) only C, (iii) both A and C. The calculated curves in Fig. 2 are obtained by the vectorial sum of individual responses of A and C. As shown in the figure, the vector sum of these responses fits closely to the case of the simultaneous illumination of $\mathrm{A}$ and $\mathrm{C}$ devices, and while the phases fit well, the magnitudes do not. This is because the measurements were done at different times and due to the difficulty of the laser alignments, the magnitudes could not be perfectly aligned to the former state. However, one should note that the normalized response in the experimental curves and the calculated curve fit very well. In the following section, we investigate the calculations in the inverse approach. That is, given the simultaneous illumination data, we extract the individual contributions of devices $\mathrm{A}$ and $\mathrm{C}$ by using the measured phase and magnitude of superposition of the two responses.

\section{Example of Extraction of the Response of Two Sensing Pixels with One Read-Out Pixel}

As shown in Eq. (2), the phase of the cross-talk-based response of the readout pixel depends on the modulation frequency of the incident radiation, the distances from the sensing pixels, and intensities of the incident radiation on the sensing pixels. The distance between pixels is constant, and a specific modulation frequency can be chosen. In this case, the phase of the readout pixel is affected only by the intensities of the incident radiation on the sensing pixels. Thus by measuring the phase of the readout pixel, the intensities of the incident radiation on the sensing pixels can be obtained. To be able to achieve this, some calibration measurements are needed to be done by radiating with various intensities on the sensing pixels.

For instance, at a $174 \mathrm{~Hz}$ modulation frequency, the following calibration measurements can be done:

- When only the far sensing pixel (C) is illuminated, the phase of the readout pixel is $-61^{\circ}$ [taking into account the leaking laser beam, $\operatorname{Mag}(\mathrm{A}) /$ $\operatorname{Mag}(\mathrm{C})=100]$.

- When the incident light on the far pixel (C) is double the near pixel (A), the phase of the readout pixel is $-40^{\circ}$.

- When both of the sensing pixels are illuminated equally, the phase of the readout pixel is $-36^{\circ}$.

- When the incident light on the near pixel (A) is double, the far pixel $(\mathrm{C})$, the phase of the readout pixel is $-34^{\circ}$.

- When only the near sensing pixel (A) is illuminated, the phase of the readout pixel is $-31^{\circ}$ [taking into account the leaking laser beam, Mag(A)/ $\operatorname{Mag}(\mathrm{C})=0.01]$.

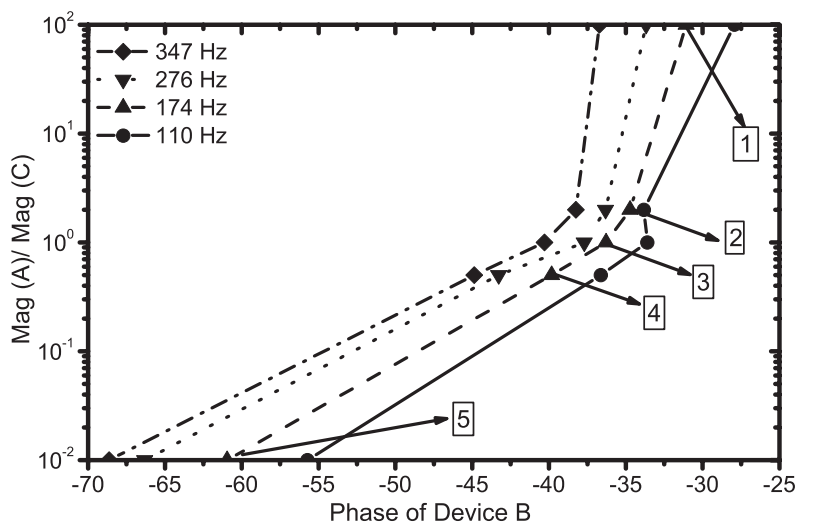

Fig. 3. $\operatorname{Mag}(\mathrm{A}) / \mathrm{Mag}(\mathrm{C})$ versus phase of readout device B. By measuring the phase of device $\mathrm{B}, \operatorname{Mag}(\mathrm{A}) / \operatorname{Mag}(\mathrm{C})$ can be obtained. The numbers in squares show the calibration data points.

As shown, when the relative incident radiation on the near sensing pixel increases, the phase of the response of the readout pixel increases. In other words, the lag of the cross-talk response decreases. When a proper modulation frequency is chosen, the ratios of the incident radiation on the sensing pixels and the phase of the readout pixel have a one-to-one relation as shown in Fig. 3. The data points can be interpolated to get the ratio of the incident radiation on the sensing pixels. In addition, by using the proposed analytical model, ${ }^{4}$ or the individual pixels response data, ${ }^{1}$ the exact values of the responses can be obtained by using the readout pixels' data.

For example, at $174 \mathrm{~Hz}$, the cross-talk response magnitude of devices $\mathrm{A}$ and $\mathrm{C}$ are 31.8 and $10.1 \mu \mathrm{V}$, respectively, as experimentally reported ${ }^{1}$ or predicted by the analytical model. 4 Thus when the A and $\mathrm{C}$ pixels are illuminated equally, the relative effect of the cross talk of these pixels on the read-out pixel is $31.8 / 10.1=3.1$. That is, the data points in Fig. 3 should be reevaluated by taking into account this ratio, which is named as $k_{1}$.

If we formulate the cross-talk response as the superposition of the responses of $\mathrm{A}$ and $\mathrm{C}$ pixels on the B pixel based on Eq. (2),

$$
V_{b} e^{-j \theta_{b}}=V_{a} e^{-j \theta_{a}}+V_{c} e^{-j \theta_{c}},
$$

where $V_{a}$ and $V_{c}$ are the magnitudes of the responses of pixels $\mathrm{A}$ and $\mathrm{C}, \theta_{a}$ and $\theta_{c}$ are the phases of the responses of pixels $\mathrm{A}$ and $\mathrm{C}$ when only pixels $\mathrm{A}$ and $\mathrm{C}$ are illuminated. $\theta_{b}$ and $V_{b}$ are the measured response phase and magnitude of the readout pixel. Above, $\theta_{a}$ and $\theta_{c}$ values are given as $-31^{\circ}$ and $-61^{\circ}$, respectively, and the desired unknowns are $V_{a}$ and $V_{c}$ in terms of $V_{b}$. A relationship between $V_{a}$ and $V_{c}$ can be defined as the following:

$$
V_{a}=k_{1} k_{2} V_{c}
$$

where $k_{1}$ is the relative effect of the cross talk of $\mathrm{A}$ and $\mathrm{C}$ pixels on the readout pixel at a given modulation frequency, and $k_{2}$ is the ratio of the radiation inten- 
sity falling on pixels $\mathrm{A}$ and $\mathrm{C}$ as found from the calibration in Fig. 3. If Eq. (4) is substituted into Eq. (3),

$$
\frac{V_{b}}{V_{c}}=\frac{k_{1} k_{2}\left(e^{-j \theta_{a}}\right)+\left(e^{-j \theta_{c}}\right)}{\left(e^{-j \theta_{b}}\right)} .
$$

For example, to get the individual response magnitudes of $\mathrm{A}$ and $\mathrm{C}$, given that the response of the readout pixel is $100 e^{-j 35^{\circ}}$ at $f_{m}=174 \mathrm{~Hz}$, we look at the calibration in Fig. 3 for $\theta=-35^{\circ}$, and we find the value of $k_{2}$, which is 2 . At $174 \mathrm{~Hz}, k_{1}, \theta_{a}$, and $\theta_{c}$, parameters are known to be constant for a specific device pattern and substrate material. If we substitute all the known values into Eq. (5), we get the following:

$$
\frac{V_{b}}{V_{c}}=\frac{2 \times 3.1\left(e^{-j 31^{\circ}}\right)+\left(e^{-j 61^{\circ}}\right)}{\left(e^{-j 34^{\circ}}\right)}=7.08+j 0.12 \cong 7.08 .
$$

Thus $V_{c}=V_{b} / 7.08$ and $V_{a}=3.1 V_{b} / 7.08$. For $V_{b}$ $=100 \mu V, V_{a}$ and $V_{c}$ values are found as 87.5 and $14.1 \mu \mathrm{V}$, respectively.

By using the calibration plot in Fig. 3, we can obtain $V_{a}$ and $V_{c}$ values for arbitrary $\theta_{b}$ values in the range of calibration. For example, for $\theta_{b}=-36^{\circ}, k_{2}$ is found to be 1 and we get $V_{b} / V_{c}=4-j 0.15 \cong 4$, and for $\theta_{b}=-40^{\circ}, k_{2}$ is found to be 0.5 and we get $V_{b} / V_{c}=2.5-j 0.11 \cong 2.5$. Note that $V_{b} / V_{c}$ in Eq. (5) is not real for arbitrary values of $\theta$ and $k$. However, as shown, the $V_{b} / V_{c}$ ratio is almost real for different intensities falling on $\mathrm{A}$ and $\mathrm{C}$ pixels. This shows too that the proposed approach is correct.

Though there are a number of parameters to be taken into account in the extraction of the individual responses, one should note that all the parameters except $k_{2}$ are constant for a fixed device pattern and modulation frequency. Since only $k_{2}$ is dependent on the incident radiation, once the device dimensions and $f_{m}$ are given, the individual magnitudes can be obtained by using only Eq. (5) and the calibration in Fig. 3, which gives the $k_{2}$ value for a desired phase of the readout pixel.

\section{Determination of Optimum Modulation Frequency Based on the Device Dimensions}

For a unique determination of the response of $\mathrm{A}$ and $\mathrm{C}$ devices, the $\operatorname{Mag}(\mathrm{A}) / \operatorname{Mag}(\mathrm{C})$ versus phase plots should have one-to-one correspondence, e.g., the curve of $110 \mathrm{~Hz}$ in Fig. 3 cannot be used for this purpose. In the lower end of the frequencies, since $L_{f}$ is greater than the device separations, devices $\mathrm{A}$ and $\mathrm{C}$ are coupled to each other as well. Based on the thermal diffusion length relation $L_{f}=(D / \pi f)^{1 / 2}$ and using the thermal diffusivity of $0.027 \mathrm{~cm}^{2} / \mathrm{s}$, the cross-talk-free $f_{m}$ between devices $\mathrm{A}$ and $\mathrm{C}$ would be 19.4 Hz. Below this frequency, we cannot differentiate the response of device $\mathrm{A}$ from device $\mathrm{C}$. Above the cross-talk-free $f_{m}$ of devices $\mathrm{C}$ and $\mathrm{B}$, which is around $500 \mathrm{~Hz}$, the response measured by the readout pixel $B$ would only be due to device A. Thus we should keep the $f_{m}$ above $20 \mathrm{~Hz}$ and below $500 \mathrm{~Hz}$. The most optimum operating frequencies for this given configuration is the frequencies that are just below the crosstalk-free $f_{m}$ between B and C. In Fig. 2, we see that the optimal operating frequency is around $250 \mathrm{~Hz}$ where the maximum phase difference between $\mathrm{A}$ and C pixels is obtained, which fits our estimations well.

\section{E. Determination of Optimum Device Layout Dimensions Based on the Modulation Frequency}

The studied bolometer array in this work was mainly designed for investigation of the interpixel cross talk of neighboring devices, rather than the noncontact measurement of the IR response. Hence extraction of the individual signals cannot be done efficiently for the studied devices. In this case, the error margins are large and the usable range of the modulation frequencies is narrow. As a design consideration, two main issues should be taken into account. First, the phases of the response caused by the individual sensing pixels should be as different as possible. This is while the cross-talk magnitudes of the responses should be as close to each other as possible. To be able to achieve both of these preferences at the same time, apart from the device separations, the different sizes of the sensing pixels should also be chosen accordingly. For example, if the distant devices are made larger in area, then the magnitude of the cross-talk response on the readout pixel would be greater. Since the phase of the response depends on the distance, it would not change considerably. In some applications, different sizes of sensing pixels might not be desired. In this case, the readout pixel shape would need to be adjusted so that the desired responses from the sensing pixels are obtained.

\section{References}

1. A. Bozbey, M. Fardmanesh, J. Schubert, and M. Banzet, "Superconductivity transition dependence of the thermal cross talk in YBCO edge transition bolometer arrays," IEEE Trans. Appl. Supercond. 16, 9-14 (2006).

2. Q. Hu and P. L. Richards, "Design analysis of a high $T_{c}$ superconducting microbolometer," Appl. Phys. Lett. 55, 2444-2446 (1989).

3. M. Fardmanesh, "Response analysis and modeling of high temperature superconductor edge transition bolometers," in High Temperature Superconductivity 2: Engineering Applications, A. Narlikar, ed. (Springer-Verlag, 2004), pp. 482-485.

4. A. Bozbey, M. Fardmanesh, J. Schubert, and M. Banzet, "Analytical modeling of the interpixel thermal cross talk in superconducting edge transition bolometer arrays," Supercond. Sci. Technol. 19, 606611 (2006). 